\title{
Biased Random Forest For Dealing With The Class Imbalance Problem
}

\author{
Mohammed Bader-El-Den Member, IEEE, Eleman Teitei and Todd Perry
}

\begin{abstract}
The class imbalance issue has been a persistent problem in machine learning that hinders the accurate predictive analysis of data in many real-world applications. The Class imbalance problem exists when the number of instances present in a class (or classes) is significantly fewer than the number of instances belonging to another class (or classes). Sufficiently recognising the minority class during classification is a problem as most algorithms employed to learn from data input are biased towards the majority class. The underlying issue is made more complex with the presence of data difficult factors embedded in such data input. This paper presents a novel and effective ensemble-based method for dealing with the class imbalance problem. This study is motivated by the idea of moving the oversampling from the data level to the algorithm level, instead of increasing the the minority instances in the datasets, the algorithms in this paper aims to âoversample the classification ensembleâ by increasing the number of classifiers that represent the minority class in the ensemble i.e. Random Forest. The proposed Biased Random Forest BRAF algorithm employs the nearest neighbour algorithm to identify the critical areas in a given dataset. The standard random forest is then fed with more random-trees generated based on the critical areas. The results show that the proposed algorithm is very effective in dealing with the class imbalance problem.
\end{abstract}

Index Terms-Class Imbalance, Classification, Random Forest, Nearest Neighbour.

\section{INTRODUCTION}

Classification algorithms have aided the analysis and prediction of data in many real-world application domains. However, learning algorithms encounter difficulties of assigning correct labels to instances when learning from imbalanced data distribution schemes, this is generally referred to as the 'class imbalance problem'. The class imbalance problem exists when a class(es) commonly referred to as the minority class(es) is under-represented when compared against the other class(es), also known as the majority class(es). Such scenario exists in many real-life applications [1], [2].

Several approaches initiated to deal with the imbalance problem ranging from externally re-balancing the class distribution to internally tweaking the learning algorithms to adapt to the imbalanced nature, have all accentuated success in varying degrees. Notwithstanding, some researchers have argued that learning algorithms adequately learn from the minority class when they are linearly separable from their majority counterparts [3]. The points at which the minority instances

Bader-El-Den and Eleman Teitei are with the School of Computing, University of Portsmouth, Buckingham Building, Lion Terrace, Portsmouth PO1 3HE, UK Email: Eleman.Teitei@ port.ac.uk Todd Perry, Research and Development, Huq Industries, 17-21 Emerald St London WC1N 3QA United Kingdom

Manuscript received N/A; revised N/A. are positioned within the majority instances in an imbalance scheme contributes to the increase in mis-classification rate thus, commonly referred to as data difficult factors [4], but will be reffered to as critical or difficult areas in this paper. These factors include, but are not limited to: small disjunts, class overlap, borderline, noise, outliers and rare instances [1].

This paper is motivated by the idea of moving the oversampling from data level to the algorithm level. In other words, instead of increasing the the minority instances, the proposed algorithms aims to "oversample the ensemble" by increasing the number of classifiers that represent the minority class in the ensemble (Random Forest in this study). Therefore, this paper introduces a hybrid ensemble method - BRAF(Biased Random Forest) - aimed at adequately representing the minority class during classification. BRAF employs the nearest neighbour algorithm to identify the difficult areas in the dataset which are the minority instances with their $k$ nearest majority neighbours. The standard random forest is then fed with more random-trees generated based on the critical or difficult areas, resulting in a more diverse ensemble/forest and at the same time biased towards the minority class. The bias in the forest aims to overcome the low presence of the minority class(es).

"Given a dataset with imbalanced classes, can extending the decision trees of a random forest to not only learn from the original training set, but also from the critical/difficult areas of a given imbalanced dataset improve the recognition of the minority instances during classification? How could these difficult areas be discovered? What is the impact of feeding these trees on the ensemble diversity? Thus, the objectives of this study are:

- Design a hybrid framework that generates an ensemble that is biased towards the minority class(es).

- Select a mechanism for defining difficult/critical areas in the input dataset.

- Evaluate and compare BRAF against other state of the art methods using several artificial and real-world binary class imbalanced datasets.

- Evaluate the diversity of the new biased forests.

The rest of the paper is structured as follows: Section II briefly explains and reviews some of the related state of the art approaches developed to tackle the class imbalance problem. Section 3 describes the proposed approach, BRAF. Section 4 encompasses various measures used in this paper for evaluating BRAF's performance and its experimental outcomes. Lastly, section 5 summarizes the paper. 


\section{BACKGROUND}

\section{A. Dealing with Imbalance Problems}

Methods employed to tackle class imbalance are generally categorised as follows: data-level approaches that aim to reduce the imbalanced data ratio by adding more minority instances (oversampling) or discarding some of the majority instances (undersampling) [5]. These techniques are mostly employed during the data pre-processing phases and are independent of the succeeding learning algorithms. Approaches at the algorithmic-level aim at internally modifying algorithms used for classification to sufficiently learn from the minority instances [6]. A detailed understanding of the corresponding algorithm and domain is required when employing methods from this category. Algorithmic approaches include, but are not limited to one class learning [7], and changing the internal bias [8], [9]. Also classified as a category is cost-sensitive learning which could be referred to as a hybrid approach, as it integrates the inclusion of cost to instances (data-level), and adjusting the succeeding training processes to accept cost (algorithmic level) [10]. Furthermore, ensemble learning methods learn from data by employing several base classifiers and systemically combining their outcome to produce a single predictive decision.

While the existing methods have emanated varying degrees of success when applied in imbalanced data schemes, this paper concentrates more on the data-level and the ensemble categories. The reason is because the motivation of BRAF's concept was deduced from both categories.

Most data-level approaches embed the NNR (Nearest Neighbour Rule) concept which emanates from the hypotheses on the role of the mutual positions of the learning instances in the feature space. Some of such data-level approaches include but are not limited to: SMOTE (Synthetic Minority Oversampling Technique). It creates synthetic minority instances from a chosen point of the line that links a selected minority instance (seed) to its nearest minority neighbour [11]. Borderline-SMOTE creates artificial instances from only those minority instances that are located near the decision border [12]. Safe-LevelSMOTE [13] and LN-SMOTE [14] generate synthetic minority instances only from examples located in safe areas. Furthermore, ADASYN(Adaptive Synthetic Sampling) generates more synthetic instances from the identified difficult regions and lesser instances from safe areas [15]. Also, ENN (Edited Nearest Neighbour) discards majority instances where their two or three nearest neighbours belong to the minority class [16]. NCR (Nearest Cleaning Rule) is similar to ENN, but further discards majority instances that surround a minority instance [17].

\section{B. Random Forest}

Ensemble learning systemically combines the learning outcomes of several base classifiers so as to deduce a single prediction that outperforms the outcome of individual base classifiers [1]. The underlying concept centres around analysing the opinion of different individuals so as to deduce a single sound opinion. An ensemble learning method's effectiveness is mainly dependent on distinctiveness of the individual base classifiers. Two popular ensemble learning techniques are Bagging (Bootstrap Aggregation) [18] and Boosting [19]. In bagging, classifiers are trained in parallel on each bootstrap sample generated from the training set. The predictive outcomes made by each classifier are systematically analysed and a single decision is deduced using majority voting. On the other hand, boosting trains classifiers in a sequential manner. Each succeeding classifier is trained on a re-weighted data subset that was misclassified in the preceding phase. A popular boosting method is AdaBoost [20].

Random forest is a learning algorithm developed by Breiman [21], which conforms to the bagging concept. It is an ensemble of decision trees; each tree representing a base classifier. Its classification process is implemented by taking a majority vote of the predictions that emanates from the individual trees trained on data subsets generated from the parent training set [22].

\section{Related Work}

Several methods that entail the modification of the random forest learning concept have been proposed to deal with the class imbalance problem. Chao Chen [23] proposed WRF (Weighed Random Forest) which embeds the cost sensitive learning concept. It assigns weight to both classes (minority and majority) of a training set; the minority instances having a larger weight. The weights are embedded in two phases. Firstly to calculate the gini criterion when finding the split of instances. Secondly, class weights are fixed in each decision tree's terminal node which aids in determining the weighted majority vote. The weighted vote of each decision tree are aggregated so as to deduce a conclusive forest prediction model.

Chao Chen [23] also introduced BRF (Balanced Random Forest) which incorporates a sampling technique. BRF was proposed to tackle the possibility that some of the generated bootstrap samples might contain fewer or none of the minority instances. The underlying idea of BRF is to systematically undersample the majority class during the generation of bootstrap samples. Furthermore, Yaya et. al. [23] proposed IBRF (Imbalanced Balanced Random Forest) that systematically combined both BRF and WRF while trying to tackle the churn prediction issue in Chinese banks. IBRF's underlying idea was aimed at harnessing the strengths of WRF and BRF. While $\mathrm{BRF}$ is more tolerant to noise and also more efficient on large imbalanced data schemes, the cost sensitive learning embeded in WRF proved to be more effective on classifiers that emanate from decision tree learning methods.

Another related concept employed to tackle class imbalance is UnderBagging [24]. UnderBagging is a combination of undersampling and bagging. The underlying idea can be implemented in two ways. Either the majority class is undersampled before bagging is applied, or it is undersampled in each of the bootstrap sample generation stages. The UnderBagging concept has been applied to varying degrees using dissimilar titles such as QuasiBagging [25], Asymmetric Bagging [26] and Roughly-balanced Bagging [27].

The minority class which is the point of interest, is always under-represented in any imbalanced data scheme. A similar 
scenario is also apparent during the random forest learning process. There exists a high probability that fewer or no minority instances will be present in the generated bootstrap samples, which in-turn, contributes to the insufficient recognition of the minority class [23].

\section{BIASED RANDOM FOREST}

This section presents the Biased Random Forest BRAF which is a simple and effective method that can be combined with any ensemble method. BRAF is motivated by the idea of moving the oversampling from data level to the algorithm level. In other words, instead of increasing the the minority instances in the dataset, the algorithm in this paper aims to "oversample the classification ensemble" by increasing the number of classifiers that represent the minority class in the ensemble. This oversampling of the classification ensemble aims to generate an ensemble biased towards the minority class to compensate its low presence in dataset.

The overall structure of the BRAF algorithm is shown in figure 1. Firstly, the nearest neighbour algorithm is employed by the BRAF algorithm to identify the difficult/critical areas in the dataset, which are the minority instances and their $k$ nearest majority neighbours. Secondly, a standard random forest is generated from the all the records in the dataset. Thirdly, the standard random forest is then fed with more random-trees generated based on the difficult areas, hopefully resulting in a more diverse ensemble/forest and at the same time, biased towards the minority class. The bias in the forest aims to overcome the low presence of instances belonging to the minority class(es).

Another interesting way of looking at BRAF, is to consider the first step in which the difficult areas are defined as an aggressive undersampling of the main data input using knearest neighbour algorithm. In this scenario, an undersampled sub-training set that contains all the minority instances and their $k$ nearest majority neighbours is generated from the original training set. Then, the random forest's decision trees are employed to learn from the bootstrap samples generated from the original training set, as well as those generated from the sub-training set.

The BRAF algorithm is detailed in Algorithm 1; given a binary imbalanced training set $T$, BRAF firstly divides the dataset into: (a) a majority set $T_{m a j}$ containing the records from the majority class(es) and (b) a minority set $T_{\min }$ containing the records from the minority class(es). Then, the potential difficult areas affecting the minority instances are defined and stored in $T_{c}$. This is done by finding the $k$ nearest majority neighbours for each record in $T_{\min }$. The $k$ nearest majority neighbours shared by two or more minority instances are extracted only once so as to avoid duplication. The decision trees of the forest are applied to not only learn from the bootstrap samples generated from $T$, but are also extended to learn from those generated from $T_{c}$; this is done by combing two separate random forests. The first forest $R F_{1}$ is generated based on the full dataset, $T$ while the second forest $R F_{s}$ is generated based on the under-sampled dataset, $T_{c}$. BRAF uses two parameters to define the size of the generated random forest. The first parameter is $s$ which defines the size of the combined forest, while the second parameter is $p$, where $0 \leq p \leq 1$ which is the ratio used to define the size of $R F_{2}$ and $R F_{1}$ i.e. the size of $R F_{2}=p * s$ and consequently the size of $R F_{1}=(1-p) * s$.

In the training phase, random forest applies the bagging concept to iteratively generate sub-training sets (bootstrap samples) with replacements. The generated bootstrap samples contain the same number of records present in their parent training sets. These records are chosen at random. An average of $64 \%$ of instances in the parent training sets are available in the bootstrap samples. Randomly choosing instances accentuates a high possibility that some instances will be repeated multiple times while some might not appear at all. This is the point where BRAF's effectiveness is accentuated as the generated bootstrap samples are likely to contain fewer or none of the minority instances. BRAF's concept serves as a boost to enhance random forest's capability to effectively recognise the minority instances in the sense that extracting bootstrap samples from $T_{c}$ (depending on the chosen $k$ parameter) increases the chances of more minority instances to be present, thereby improving the accuracy of the outcome of the individual trees. This is controlled by the parameter $p$; high value of $p$ increases the number of decision trees learning from the difficult/critical areas $T_{c}$ or decreases the number of those learning from the full training set $T$. The prediction of each individual tree is systematically analysed to produce a single outcome by applying majority voting. The built classification model is further used to assign labels to unlabelled instances belonging to the test set $T s$.

\section{EXPERIMENTAL STUDY}

This section shows the performance of the proposed BRAF method. The aim of this section is:

1) Analyse the performance of BRAF using different parameters i.e. $k$ number of neighbours and $p$ ratio of difficult areas $T_{c}$.

2) Show the performance gain of BRAF when compared to the standard assemble methods.

3) To compare the performance of BRAF with similar methods, in particular, data-level oversampling methods (BRAF could be seen as method for over sampling the assignable instead oversampling the data).

4) Analyse the diversity of the proposed BRAF methods, as diversity has been used to explain the success of several ensemble methods, theoretically and practically [28].

\section{A. Evaluative Performance Metrics}

In classification, accuracy is commonly used as a performance metric for measuring the percentage of correct predictions made. Accuracy has however proven not to be a suitable metric to be exerted in a class imbalance scenario as it is biased towards the majority instances and maintains a high percentage of accuracy even when all the minority examples are mis-classified. Being that the minority class is the point of interest, other performance measurement metrics such as ROC (Receiver Operating Characteristic), AUC (Area Under Curve), F-measure and G-mean which is adopted in this paper. 


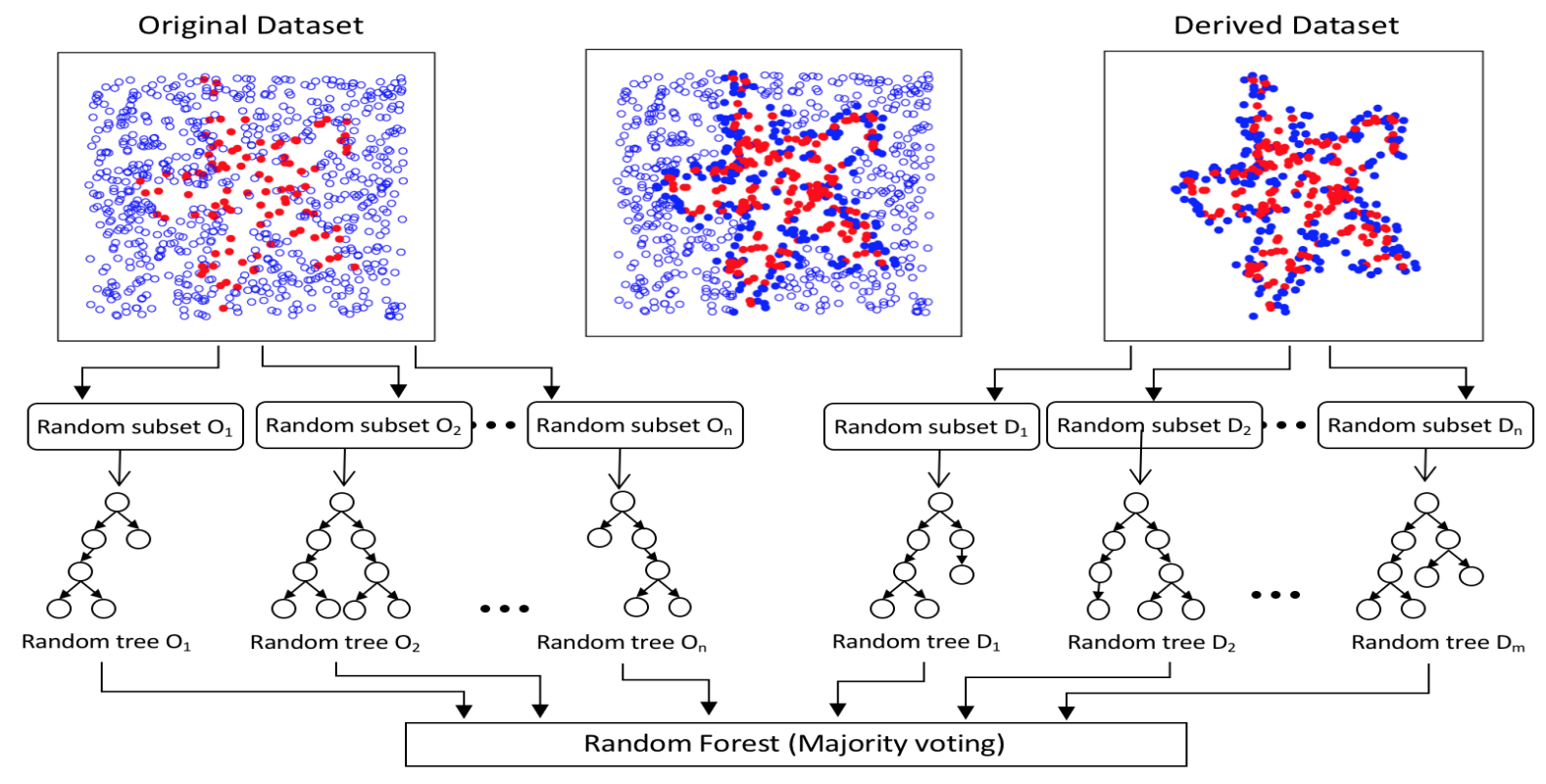

Fig. 1. Pictorially illustrates BRAF's concept for a binary class imbalance scheme. The difficult/critical areas of the Subclus 70 dataset are identified using $k=5$ when defining the nearest majority neighbour parameter. Bootstrap samples are generated from the original training set, $T$ as well as the derived training set, $T_{c}$. The random trees of the forest are then employed to not only learn from $T$, but also extended to learn from the derived data input $T_{c}$

\section{B. Datasets}

Experiments were carried out using 21 imbalanced binary datasets. 12 of these datasets are artificial datasets from the KEEL repository [29]. They all have 800 examples with an imbalance ratio of 1:7. Their majority instances were invariably spread around the minority instances taking the shapes of a paw, several sub-clusters, and a clover as depicted in figure 2. The minority instances in the paw dataset were decomposed into three elliptic smaller-regions with two of its regions situated near each other. The minority instances in the clover dataset are organized in an order that portrays a flower with elliptic petals which make them non-linear and more difficult for an algorithm to learn from. While in the sub-cluster dataset, minority instances are placed in rectangular shapes which are uniformly surrounded by the majority instances. To increase their difficulty, the disturbance ratio of their underlying borderline and small disjunt examples were increased by $30 \%, 50 \%$ and $70 \%$ respectively.

Furthermore, 9 real-world application datasets were downloaded from the UCI repository [30]. These datasets have varying degrees of imbalance ratios, and contain few or no safe minority examples [31]. For example, the minority examples in the Herbaman dataset are made up of 10 safe, 21 outlier and 51 borderline instances. See Table I for their description, including the number of instances, number of features, and imbalance ratio (IR)

\section{Experimental Setup and Analysis}

In order to analyse and understand the performance of the proposed BRAF method, different sets of experiments were carried out, where the performance of the BRAF was compared against the performance of some other state of the art methods. Also, we tested BRAF (with and without
TABLE I

DESCRIPTION OF THE BINARY IMBALANCED DATASETS USED IN THIS PAPER

\begin{tabular}{|c|c|c|c|c|c|c|}
\hline & Dataset & \#Instances & \#Features & \# Min & \#Maj & \#IR \\
\hline \multirow{12}{*}{ 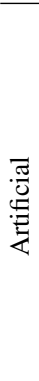 } & Clover0 & 800 & 2 & 100 & 700 & 7 \\
\hline & Clover30 & 800 & 2 & 100 & 700 & 7 \\
\hline & Clover50 & 800 & 2 & 100 & 700 & 7 \\
\hline & Clover70 & 800 & 2 & 100 & 700 & 7 \\
\hline & Paw0 & 800 & 2 & 100 & 700 & 7 \\
\hline & Paw30 & 800 & 2 & 100 & 700 & 7 \\
\hline & Paw50 & 800 & 2 & 100 & 700 & 7 \\
\hline & Paw70 & 800 & 2 & 100 & 700 & 7 \\
\hline & Subclus0 & 800 & 2 & 100 & 700 & 7 \\
\hline & Subclus30 & 800 & 2 & 100 & 700 & 7 \\
\hline & Subclus50 & 800 & 2 & 100 & 700 & 7 \\
\hline & Subclus70 & 800 & 2 & 100 & 700 & 7 \\
\hline \multirow{9}{*}{ 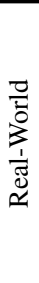 } & Abalone19 & 4174 & 8 & 32 & 4142 & 129.44 \\
\hline & Breast & 286 & 9 & 85 & 201 & 2.36 \\
\hline & Bupa & 345 & 8 & 145 & 200 & 1.38 \\
\hline & Car-good & 1728 & 6 & 69 & 1659 & 24.04 \\
\hline & Haberman & 306 & 19 & 81 & 225 & 2.78 \\
\hline & Hepatitis & 155 & 9 & 32 & 123 & 5.35 \\
\hline & Pima & 768 & 8 & 268 & 500 & 1.87 \\
\hline & Poker & 2075 & 10 & 25 & 2250 & 82 \\
\hline & Yeast & 1484 & 8 & 51 & 1433 & 28.1 \\
\hline
\end{tabular}

SMOTE) using different $k$ parameters. Furthermore, in order to understand the behaviour of the BRAF, diversity analysis was also carried out. In this analysis, we measured BRAF's level of diversity between the decision trees as compared against a base random forest algorithm.

In all experiments, the algorithms were run using 10 fold cross-validation. The knn employed ranged between $1-50$, while 100 decision trees were interchangeably used to learn from each of the training sets. Each experiment was run 10 times, using 21 datasets. Geometric Mean (GM) and FMeasure (FM) were used to evaluate the classification perfor- 


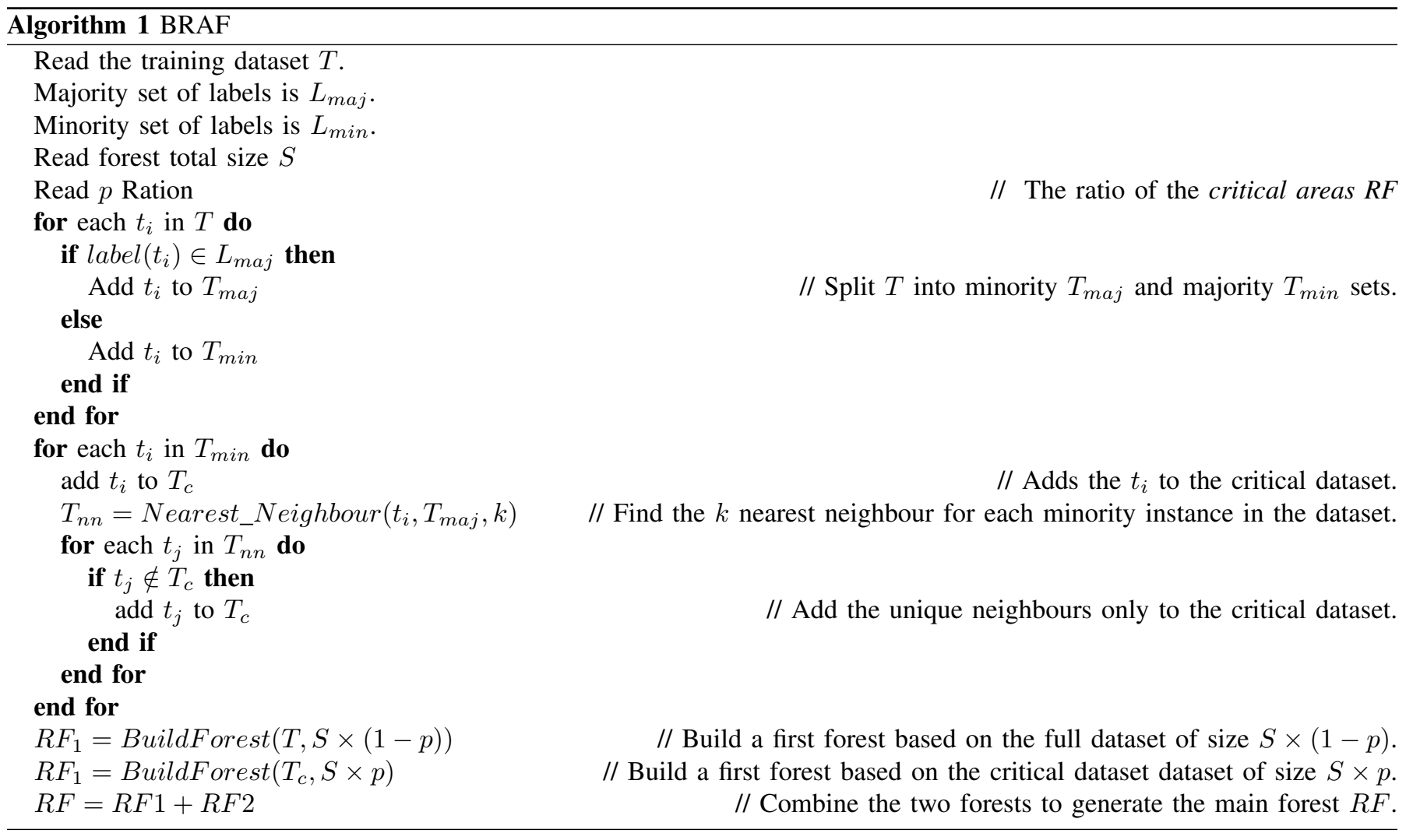

mance. Furthermore, BRAF's diversity was evaluated using the dis-agreement measure [32]. The base random forest classifier parameters was tuned by evaluating the RF parameters, the same parameters have been used for both the RF and BRAF.

1) Performance Analysis: Table II shows the performance comparison between BRAF's $k$ values and the random forest base classifier. BRAF's results were generated using the size of the forest $s=100$ and $k=1,10$ and 20. In all the experiments $p=0.50$, which means that half of the forest is build based on the original training data $T$, while the second half is based of the difficult areas dataset $T_{c}$. G-mean and F-measure are used as evaluative measurement metrics. The results depicted are the average of 10 runs \pm standard deviation. The best overall results for each dataset are marked with (*). The aim of this experiment is to ascertain the effectiveness of different $K s$ applied in BRAF when selecting the nearest majority neighbours for the difficult areas set. A total of 19 datasets were used; 7 real-world and 12 artificial datasets from the KEEL and UCI repositories respectively.

BRAF performed better when compared against the random forest base classifier across all datasets when using Fmeasure as a performance metric. However, when using Gmean, random forest performed slightly better that BRAF on Breast, Clover0, Haberman and Pima datasets.

As mentioned earlier, the target of the experiments is not to show that BRAF outperform other state-of-the-art methods, instead the target is to (1) show that it is possible to to improve the performance of an assignable on imbalanced data sets by making it biased towards the minority class. (2) to compare the performance of BRAF with similar methods, in particular, data-level oversampling methods (BRAF could be seen as method for over sampling the assignable instead oversampling the data). In order to achieve the second point, the BRAF was compared with SMOTE as it is a widely used over sampling method. SMOTE, as any sampling method is not a stand alone classifier, but it can be used in conjunction with any classifier. Table III compares the performance of BRAF (with and without SMOTE) against the base classifier RF with SMOTE. Several configurations of SMOTE were tested and best settings were used for both RF and BRAF. Unlike RF, and as expected, BRAF did not perform well when combined with SMOTE as the data became more balanced. Moreover, as shown in table III the performance of BRAF decreases with the increase of the number of neighbours $k$. However, the performance of the BRAF without SMOTE is very competitive and has outperformed RF+SMOTE on several occasions, specially on the GM measure.

BRAF's best results were also compared against other state of the art methods that have proven to improve classification performance in difficult class imbalance scenarios. They include include, SMOTE [11], TempC [33] and AdaBoost [20]. The experiment was carried out on 9 real-world application datasets using G-mean as evaluative performance metric. BRAF's best results were selected from $K=1-50$. As can be deduced from Table IV, BRAF performed better than the random forest base classifier, SMOTE and AdaBoost on all datasets except on Breast where AdaBoost performed slightly better. TempC was slightly competitive against BRAF as it performed better on the Bupa, Car and Yeast datasets.

As shown in the results, the $k$ value could have an impact on the and performance of BRAF may vary from one dataset to another because, different datasets may have different 
(a) Clover70 - Original set

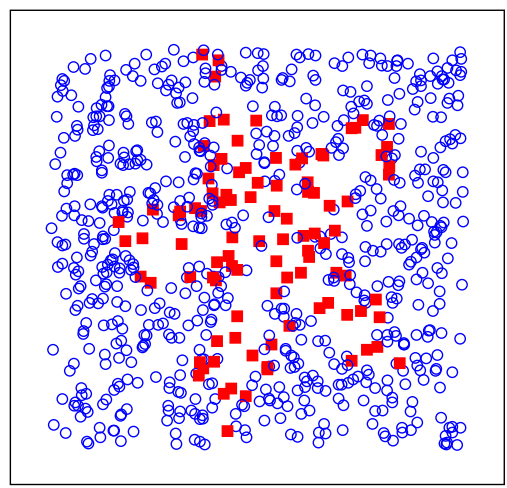

(d) $k=10$

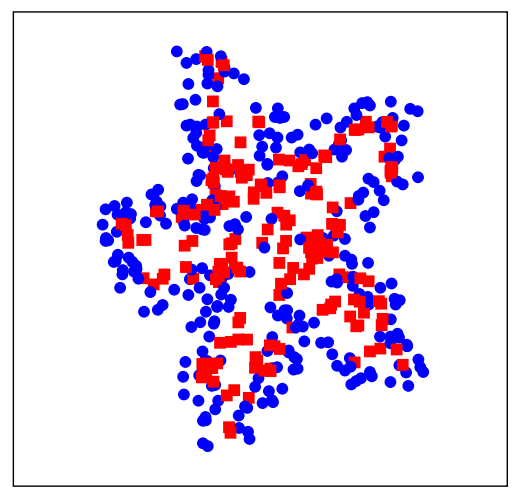

(g) Paw70 - Original set

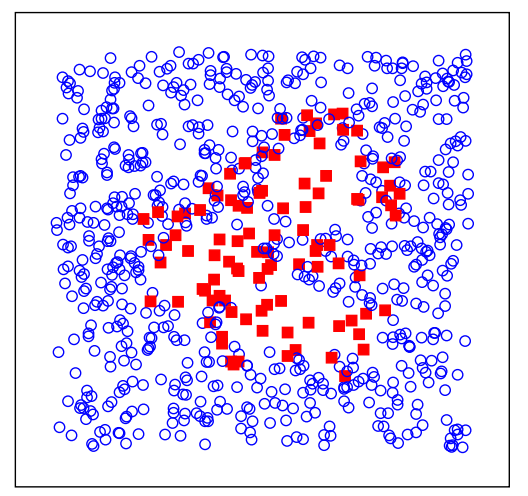

(j) $k=10$

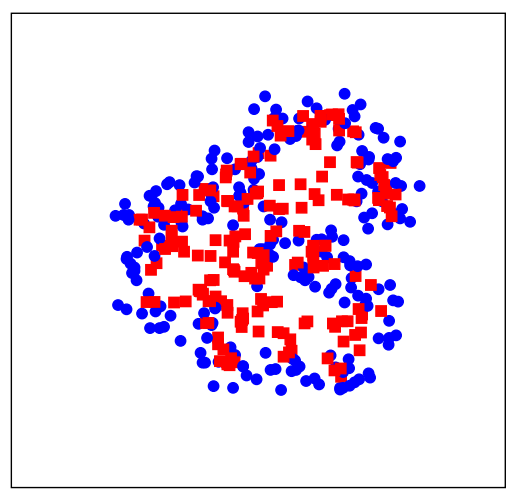

(b) $k=1$

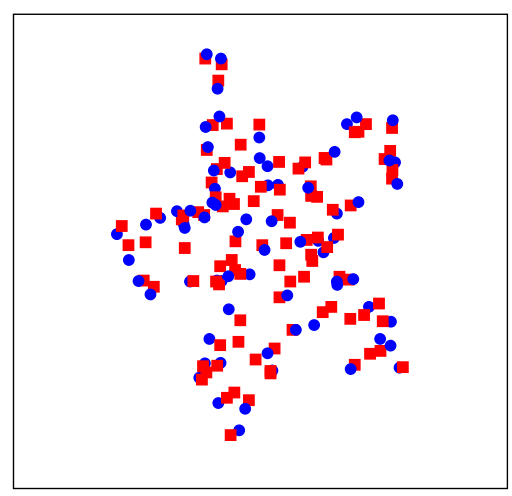

(e) $k=20$

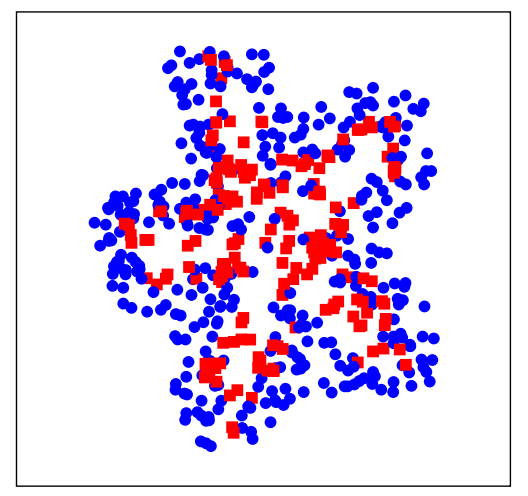

(h) $k=1$

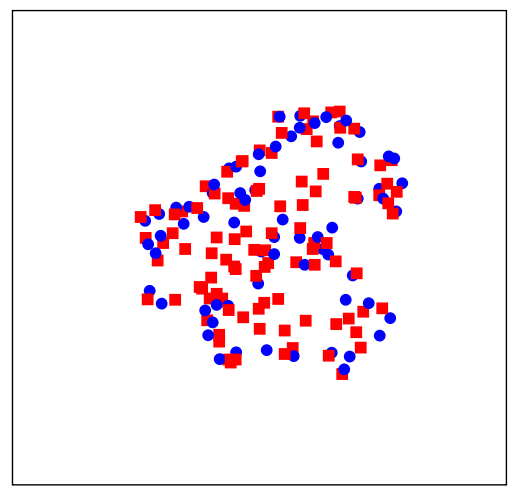

(k) $k=20$

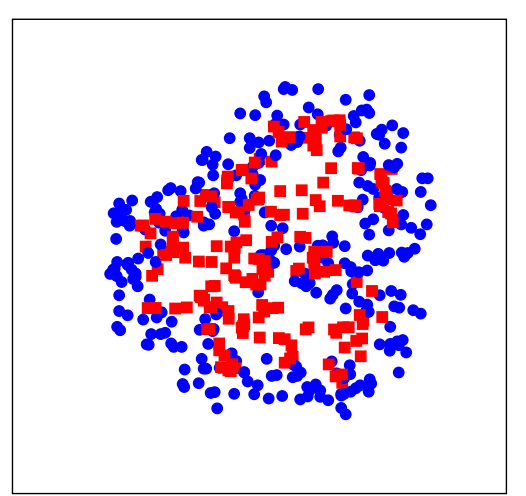

(c) $k=5$

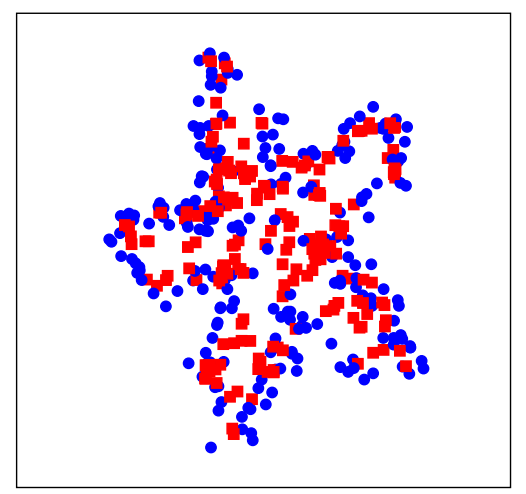

(f) $k=50$

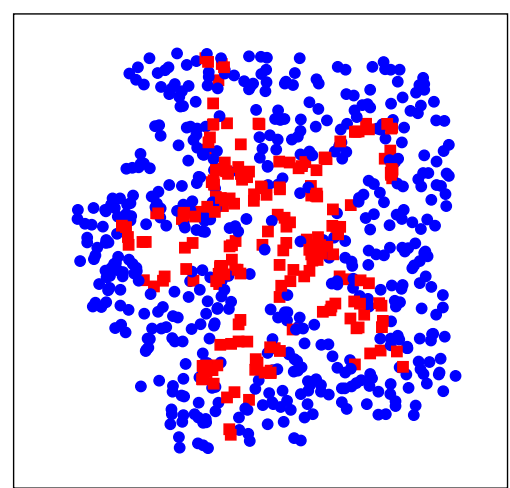

(i) $k=5$

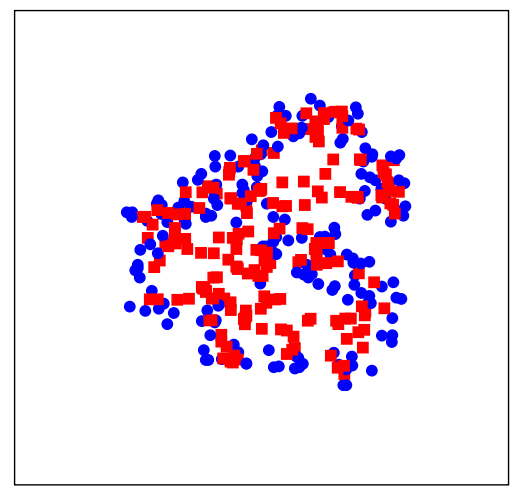

(1) $k=50$

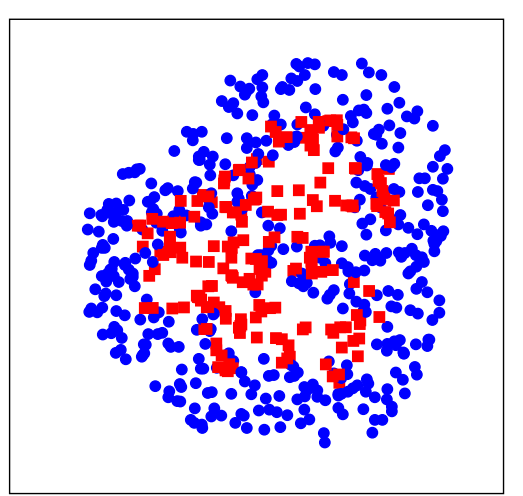

Fig. 2. Visualises the impact of the number of majority neighbours $(k=\{1,5,10,20,50\})$ on the dataset. the figures shows the Colver70 and Paw70 as these are artificial datasets with only 2 attributes which make it visualisable. The $x$ and $y$ axises are the value of the first and second attributes respectively. 
TABLE II

COMPARES BRAF'S PERFORMANCE ON A RANGE OF DATASETS USING $k=1,10$ AND 20 AGAINST THE STANDARD RANDOM FOREST ALGORITHM USING G.MEAN AND F-MEASURE AS EVALUATIVE PERFORMANCE METRICS. THE RESULTS ARE THE AVERAGE OF 10 RUNS OF 10 CROSS VALIDATION, \pm THE STANDARD DEVIATION. THE BEST OVERALL RESULTS FOR EACH DATASET IS MARKED BY $(*)$

\begin{tabular}{|c|c|c|c|c|c|c|c|c|}
\hline & \multicolumn{2}{|c|}{ Base } & \multicolumn{2}{|c|}{ BRAF1 } & \multicolumn{2}{|c|}{ BRAF10 } & \multicolumn{2}{|c|}{ BRAF20 } \\
\hline & $\mathrm{fm}$ & $\mathrm{gm}$ & $\mathrm{fm}$ & $\mathrm{gm}$ & $\mathrm{fm}$ & $\mathrm{gm}$ & $\mathrm{fm}$ & $\mathrm{gm}$ \\
\hline Breast & $0.39 \pm 0.17$ & $0.60 \pm 0.14 *$ & $0.40 \pm \mathbf{0 . 1 8}^{*}$ & $0.56 \pm 0.13$ & $0.38 \pm 0.19$ & $0.59 \pm 0.15$ & $0.38 \pm 0.19$ & $0.58 \pm 0.14$ \\
\hline Bupa & $0.60 \pm 0.12$ & $0.69 \pm 0.08$ & $0.63 \pm \mathbf{0 . 1 0}$ & $0.67 \pm 0.07$ & $\mathbf{0 . 6 3} \pm \mathbf{0 . 1 0}$ & $0.71 \pm 0.05^{*}$ & $\mathbf{0 . 6 2} \pm \mathbf{0 . 1 0}$ & $0.70 \pm 0.06$ \\
\hline Car & $0.42 \pm 0.20$ & $0.74 \pm 0.29$ & $0.58 \pm 0.16^{*}$ & $\mathbf{0 . 8 3} \pm \mathbf{0 . 1 2}$ & $\mathbf{0 . 5 5} \pm \mathbf{0 . 1 5}$ & $0.84 \pm 0.14$ & $\mathbf{0 . 5 7} \pm \mathbf{0 . 1 2}$ & $0.84 \pm 0.12^{*}$ \\
\hline Clover0 & $0.59 \pm 0.11$ & $0.93 \pm \mathbf{0 . 0 8} *$ & $\mathbf{0 . 8 3} \pm \mathbf{0 . 0 7 *}$ & $0.88 \pm 0.07$ & $\mathbf{0 . 8 1} \pm \mathbf{0 . 0 8}$ & $0.91 \pm 0.07$ & $\mathbf{0 . 8 0} \pm \mathbf{0 . 0 7}$ & $0.91 \pm 0.07$ \\
\hline Clover30 & $0.60 \pm 0.09$ & $0.64 \pm 0.80$ & $0.59 \pm 0.11$ & $\mathbf{0 . 7 5} \pm \mathbf{0 . 1 0}$ & $0.60 \pm 0.11$ & $\mathbf{0 . 8 1} \pm \mathbf{0 . 0 9}$ & $0.64 \pm 0.13^{*}$ & $0.83 \pm 0.10 *$ \\
\hline Clover50 & $0.47 \pm 0.12$ & $0.75 \pm 0.13$ & $0.43 \pm 0.10$ & $0.64 \pm 0.12$ & $0.48 \pm 0.09 *$ & $0.74 \pm 0.11$ & $0.48 \pm 0.11$ & $0.75 \pm \mathbf{0 . 0 9} *$ \\
\hline Clover70 & $0.30 \pm 0.08$ & $0.61 \pm 0.13$ & $0.39 \pm \mathbf{0 . 1 1} *$ & $0.59 \pm 0.11$ & $0.28 \pm 0.08$ & $0.62 \pm \mathbf{0 . 1 2} *$ & $0.29 \pm 0.09$ & $0.62 \pm \mathbf{0 . 1 3}$ \\
\hline Haberman & $0.39 \pm 0.15$ & $0.58 \pm 0.15^{*}$ & $0.42 \pm 0.12 *$ & $0.53 \pm 0.12$ & $0.34 \pm 0.18$ & $0.51 \pm 0.22$ & $0.37 \pm 0.14$ & $0.56 \pm 0.14$ \\
\hline Hepatitis & $0.42 \pm 0.33$ & $0.57 \pm 0.41$ & $0.56 \pm \mathbf{0 . 3 2} *$ & $\mathbf{0 . 6 3} \pm \mathbf{0 . 3 4}$ & $\mathbf{0 . 5 2} \pm \mathbf{0 . 3 1}$ & $0.66 \pm \mathbf{0 . 3 6} *$ & $0.37 \pm 0.35$ & $0.51 \pm 0.44$ \\
\hline Paw0 & $0.91 \pm 0.08$ & $0.94 \pm 0.05$ & $0.90 \pm 0.1$ & $0.94 \pm 0.07$ & $0.92 \pm 0.07 *$ & $0.96 \pm \mathbf{0 . 0 4} *$ & $0.91 \pm 0.07$ & $\mathbf{0 . 9 5} \pm \mathbf{0 . 0 4}$ \\
\hline Paw30 & $0.65 \pm 0.15$ & $0.40 \pm 0.07$ & $\mathbf{0 . 6 8} \pm \mathbf{0 . 1 5}$ & $\mathbf{0 . 8 0} \pm \mathbf{0 . 1 1}$ & $0.69 \pm 0.13$ & $\mathbf{0 . 8 5} \pm \mathbf{0 . 0 8 *}$ & $0.71 \pm 0.15 *$ & $0.84 \pm \mathbf{0 . 0 9}$ \\
\hline Paw50 & $0.57 \pm 0.09$ & $0.78 \pm 0.08$ & $0.59 \pm 0.09$ & $0.75 \pm 0.09$ & $0.59 \pm \mathbf{0 . 0 9}$ & $0.79 \pm \mathbf{0 . 0 8}$ & $0.63 \pm 0.07 *$ & $\mathbf{0 . 8 2} \pm \mathbf{0 . 0 7 *}$ \\
\hline Paw70 & $0.44 \pm 0.17$ & $0.74 \pm 0.15$ & $0.49 \pm 0.12 *$ & $0.69 \pm 0.10$ & $\mathbf{0 . 4 7} \pm \mathbf{0 . 1 5}$ & $\mathbf{0 . 7 5} \pm \mathbf{0 . 1 5}$ & $0.49 \pm 0.14$ & $\mathbf{0 . 7 7} \pm \mathbf{0 . 1 4} *$ \\
\hline Pima & $0.21 \pm 0.07$ & $0.76 \pm \mathbf{0 . 0 4} *$ & $0.66 \pm \mathbf{0 . 0 8}$ & $0.71 \pm 0.06$ & $\mathbf{0 . 6 6} \pm \mathbf{0 . 0 7}$ & $0.74 \pm 0.04$ & $0.67 \pm 0.07 *$ & $0.75 \pm 0.04$ \\
\hline Poker & $0.00 \pm 0.00$ & $0.00 \pm 0.00$ & $0.47 \pm \mathbf{0 . 4 3} *$ & $0.60 \pm 0.51 *$ & $0.21 \pm \mathbf{0 . 3 6}$ & $\mathbf{0 . 3 0} \pm \mathbf{0 . 4 8}$ & $\mathbf{0 . 0 4} \pm \mathbf{0 . 1 3}$ & $\mathbf{0 . 1 0} \pm \mathbf{0 . 3 1}$ \\
\hline Subc0 & $0.96 \pm 0.06$ & $0.98 \pm 0.03$ & $0.96 \pm 0.06$ & $0.98 \pm 0.03$ & $0.96 \pm 0.06$ & $0.98 \pm 0.03$ & $0.96 \pm 0.06$ & $0.98 \pm 0.03$ \\
\hline Sub30 & $0.69 \pm 0.07$ & $0.87 \pm 0.06$ & $0.68 \pm 0.1$ & $0.83 \pm 0.10$ & $\mathbf{0 . 7} \pm \mathbf{0 . 0 7 *}$ & $\mathbf{0 . 8 8} \pm \mathbf{0 . 0 5 *}$ & $0.69 \pm 0.08$ & $\mathbf{0 . 8 8} \pm \mathbf{0 . 0 7}$ \\
\hline Subc50 & $0.41 \pm 0.14$ & $0.68 \pm 0.13$ & $0.41 \pm 0.11 *$ & $0.68 \pm 0.12$ & $0.40 \pm 0.13$ & $0.69 \pm \mathbf{0 . 1 1} *$ & $0.41 \pm \mathbf{0 . 1 2} *$ & $0.68 \pm 0.12$ \\
\hline Subc70 & $0.30 \pm 0.12$ & $0.59 \pm 0.14$ & $0.44 \pm \mathbf{0 . 1 2}$ & $0.61 \pm 0.09$ & $\mathbf{0 . 4 0} \pm \mathbf{0 . 1 4}$ & $0.61 \pm 0.13$ & $0.40 \pm 0.11 *$ & $0.62 \pm 0.09 *$ \\
\hline
\end{tabular}

TABLE III

COMPARES BRAF'S PERFORMANCE USING DIFFERENT $k^{\prime} s-k=1,10$ AND 20- AGAINST THE SMOTE + STANDARD RANDOM FOREST ALGORITHM AND SMOTE + BRAF USING G.MEAN AND FMEASURE AS EVALUATIVE PERFORMANCE METRICS. THE RESULTS ARE THE AVERAGE OF 10 RUNS \pm THE STANDARD DEVIATION. THE BEST OVERALL RESULTS FOR EACH DATASET IS MARKED BY $(*)$

\begin{tabular}{|c|c|c|c|c|c|c|c|c|}
\hline & \multicolumn{2}{|c|}{ Base + SMOTE } & \multicolumn{2}{|c|}{ BRAF1 + SMOTE } & \multicolumn{2}{|c|}{ BRAF10 + SMOTE } & \multicolumn{2}{|c|}{ BRAF10 } \\
\hline & FM & GM & FM & GM & FM & GM & FM & GM \\
\hline Breast & $0.43 \pm 0.14$ & $0.56 \pm 0.11$ & $0.45 \pm \mathbf{0 . 1 3}$ & $0.56 \pm 0.13$ & $0.49 \pm 0.14 *$ & $0.59 \pm \mathbf{0 . 1 5}$ & $0.38 \pm 0.19$ & $0.59 \pm \mathbf{0 . 1 5}$ \\
\hline Bupa & $0.63 \pm 0.09$ & $0.67 \pm 0.06$ & $0.58 \pm 0.08$ & $0.02 \pm 0.12$ & $0.50 \pm 0.10$ & $0.45 \pm 0.21$ & $0.63 \pm 0.1^{*}$ & $0.71 \pm \mathbf{0 . 0 5 *}$ \\
\hline Car & $0.66 \pm 0.11 *$ & $0.83 \pm 0.08$ & $0.58 \pm 0.16$ & $0.83 \pm 0.12$ & $0.55 \pm 0.15$ & $0.84 \pm 0.14$ & $0.55 \pm 0.15$ & $0.84 \pm \mathbf{0 . 1 4} *$ \\
\hline Clover0 & $0.84 \pm \mathbf{0 . 0 9}$ & $\mathbf{0 . 9 3} \pm \mathbf{0 . 0 7}$ & $0.25 \pm 0.07$ & $0.30 \pm 0.17$ & $0.20 \pm 0.11$ & $0.30 \pm 0.15$ & $0.81 \pm 0.08$ & $0.91 \pm 0.07$ \\
\hline Clover30 & $0.69 \pm 0.09 *$ & $0.79 \pm 0.08$ & $0.04 \pm 0.03$ & $0.02 \pm 0.05$ & $0.00 \pm 0.00$ & $0.00 \pm 0.00$ & $0.60 \pm 0.11$ & $0.81 \pm 0.09 *$ \\
\hline Clover50 & $0.56 \pm 0.11 *$ & $0.72 \pm 0.12$ & $0.25 \pm 0.07$ & $0.33 \pm 0.14$ & $0.00 \pm 0.03$ & $0.01 \pm 0.04$ & $0.48 \pm 0.09$ & $0.74 \pm \mathbf{0 . 1 1 *}$ \\
\hline Clover70 & $0.42 \pm 0.09 *$ & $0.64 \pm 0.1^{*}$ & $0.24 \pm 0.07$ & $0.22 \pm 0.20$ & $0.00 \pm 0.00$ & $0.00 \pm 0.00$ & $0.28 \pm 0.08$ & $0.62 \pm 0.12$ \\
\hline Haberman & $0.40 \pm 0.17$ & $\mathbf{0 . 5 4} \pm \mathbf{0 . 1 5 *}$ & $\mathbf{0 . 4 5} \pm \mathbf{0 . 1 0}$ & $0.45 \pm 0.12$ & $\mathbf{0 . 5 5} \pm \mathbf{0 . 1 0 *}$ & $0.51 \pm 0.22$ & $0.34 \pm 0.18$ & $0.51 \pm 0.22$ \\
\hline Hepatitis & $0.45 \pm 0.36$ & $0.52 \pm 0.38$ & $0.39 \pm 0.18$ & $0.44 \pm 0.21$ & $0.36 \pm 0.21$ & $0.47 \pm 0.24$ & $0.52 \pm \mathbf{0 . 3 1} *$ & $0.66 \pm 0.36 *$ \\
\hline Paw0 & $0.90 \pm 0.08$ & $0.94 \pm 0.05$ & $0.27 \pm 0.06$ & $0.39 \pm 0.06$ & $0.28 \pm 0.07$ & $0.40 \pm 0.06$ & $\mathbf{0 . 9 2} \pm \mathbf{0 . 0 7} *$ & $0.96 \pm 0.04 *$ \\
\hline Paw30 & $0.68 \pm 0.12$ & $0.40 \pm 0.08$ & $0.26 \pm 0.07$ & $0.00 \pm 0.00$ & $0.00 \pm 0.00$ & $0.85 \pm 0.08$ & $\mathbf{0 . 6 9} \pm \mathbf{0 . 1 3} *$ & $\mathbf{0 . 8 5} \pm \mathbf{0 . 0 8}^{*}$ \\
\hline Paw50 & $0.62 \pm 0.05^{*}$ & $0.75 \pm 0.06$ & $0.25 \pm 0.07$ & $0.31 \pm 0.16$ & $0.20 \pm 0.11$ & $0.30 \pm 0.15$ & $0.59 \pm 0.09$ & $0.79 \pm 0.08 *$ \\
\hline Paw70 & $0.53 \pm \mathbf{0 . 1 3} *$ & $0.71 \pm 0.09$ & $0.13 \pm 0.06$ & $0.12 \pm 0.14$ & $0.00 \pm 0.00$ & $0.00 \pm 0.00$ & $0.47 \pm 0.15$ & $0.75 \pm \mathbf{0 . 1 5 *}$ \\
\hline Pima & $0.69 \pm \mathbf{0 . 0 6 *}$ & $0.74 \pm \mathbf{0 . 0 3}^{*}$ & $0.51 \pm 0.06$ & $0.00 \pm 0.00$ & $0.59 \pm 0.08$ & $0.67 \pm 0.05$ & $0.66 \pm 0.07$ & $0.74 \pm 0.04$ \\
\hline Poker & $0.09 \pm 0.19$ & $0.20 \pm 0.03$ & $0.03 \pm 0.03$ & $0.07 \pm 0.09$ & $0.00 \pm 0.00$ & $0.00 \pm 0.00$ & $\mathbf{0 . 2 1} \pm \mathbf{0 . 3 6} *$ & $0.30 \pm \mathbf{0 . 4 8 *}$ \\
\hline Subc0 & $0.96 \pm 0.05$ & $0.98 \pm 0.11$ & $0.31 \pm 0.07$ & $0.43 \pm 0.06$ & $0.31 \pm 0.07$ & $0.43 \pm 0.06$ & $0.96 \pm 0.06$ & $\mathbf{0 . 9 8} \pm \mathbf{0 . 0 3}^{*}$ \\
\hline Sub30 & $0.67 \pm 0.10$ & $0.82 \pm 0.07$ & $0.24 \pm 0.06$ & $0.37 \pm 0.06$ & $0.05 \pm 0.10$ & $0.07 \pm 0.15$ & $\mathbf{0 . 7 0} \pm \mathbf{0 . 0 7} *$ & $\mathbf{0 . 8 8} \pm \mathbf{0 . 0 5}^{*}$ \\
\hline Subc50 & $0.45 \pm 0.07 *$ & $0.67 \pm 0.10$ & $0.23 \pm 0.06$ & $0.33 \pm 0.11$ & $0.00 \pm 0.00$ & $0.00 \pm 0.00$ & $0.40 \pm 0.13$ & $0.69 \pm 0.11^{*}$ \\
\hline Subc70 & $0.40 \pm 0.13$ & $0.61 \pm 0.32$ & $0.24 \pm 0.07$ & $0.32 \pm 0.13$ & $0.00 \pm 0.00$ & $0.00 \pm 0.00$ & $0.40 \pm 0.14$ & $0.61 \pm \mathbf{0 . 1 3} *$ \\
\hline
\end{tabular}

landscapes. In order to demonstrate this, figure 2 pictorially illustrates the impact of $k$ on two datasets; Colver70 and Paw70. These datasets were selected as both are artificial datasets with 2 attributes each which makes it is easier to visualise. Based on the experiments in this study, the default recommended values for our BRAF method are $(k=10$ and $p=0.5)$. Also, the imbalance ratio between the majority and minority labels should be taken in consideration when selecting the $k$ value, as in SMOTE and other oversampling methods, it might be useful to increase the $k$ value with the increase of the imbalance ration to compensate the difference between the labels. Moreover, BRAF could be easily combined with almost any parameter tuning and hyperparameters optimisation methods suitable for Random Forest in particular or machine learning in general [34], [10].

2) Execution time analysis: This section provides a computational cost analysis of the proposed BRAF approach in comparison with SMOTE and RF as shown in table V. The results are in milliseconds and based on "system time" which is not very accurate but gives a good indication of the execution time. Also, the results are the average of 100 runs to compensate the inaccuracy of the method. A number of neighbours $k$ os 1 is used for both BRAF and SMOTE. BRAF training time is similar to SMOTE but slightly higher than RF as expected. In respect to the testing/operational time which is the most important factor as training is done offline, BRAF and RF testing times are almost identical as shown in table V, this is because BRAF generates a set forest of random trees 
TABLE IV

A COMPARISON OF BRAF'S PERFORMANCE AGAINST THE STANDARD RANDOM FOREST ALGORITHM, TEMPC, SMOTE AND ADABOOST USING GMEAN AS EVALUATIVE MEASUREMENT METRIC. THE BEST OVERALL RESULTS FOR EACH DATASET IS MARKED BY $(*)$.

\begin{tabular}{llrrr}
\hline Datasets & RFBase & \multicolumn{1}{c}{ BRAF } & TempC & AdaBoost \\
\hline Abalone & 0.0000 & $\mathbf{0 . 1 2 5 6}$ & 0.0000 & 0.0000 \\
Breast & 0.5962 & 0.6004 & 0.6281 & $\mathbf{0 . 6 7 6 9 *}$ \\
Bupa & 0.6913 & 0.7149 & $\mathbf{0 . 7 3 7 7 *}^{*}$ & 0.6185 \\
Car & 0.7406 & 0.8398 & $\mathbf{0 . 8 6 5 6}^{*}$ & 0.5552 \\
Haberman & 0.5741 & $\mathbf{0 . 6 8 8 8}^{*}$ & 0.5558 & 0.5388 \\
Hepatitis & 0.5741 & $\mathbf{0 . 6 7 8 3}^{*}$ & 0.6132 & 0.5249 \\
Pima & 0.7553 & $\mathbf{0 . 7 5 7 3}^{*}$ & 0.7517 & 0.6948 \\
Poker & 0.0000 & $\mathbf{0 . 5 9 8 2}^{*}$ & 0.3989 & 0.0000 \\
Yeast & 0.7953 & 0.8001 & $\mathbf{0 . 8 4 0 8 *}$ & 0.4520 \\
\hline
\end{tabular}

as in RF and SMOTE.

TABLE V

THE TESTING AND TRAINING EXECUTION TIME ANALYSIS OF BRAF IN COMPARISON WITH SMOTE AND RF. THE RESULTS ARE IN MILLISECONDS

\begin{tabular}{lllllll}
\hline & BRF & & SMOTE & RF & \\
& Test & Train & Test & Train & Test & Train \\
\hline clover0 & 64.1 & 3.2 & 60.4 & 3.1 & 51.4 & 3.1 \\
clover30 & 75.1 & 3.3 & 67.1 & 3.6 & 55.3 & 3.4 \\
clover50 & 77.7 & 3.9 & 71.9 & 3.8 & 59.2 & 3.6 \\
clover70 & 69.4 & 4.1 & 75.4 & 4.1 & 61.8 & 3.9 \\
paw0 & 47.6 & 2.1 & 48.1 & 2.2 & 39.6 & 2.0 \\
paw30 & 70.0 & 4.1 & 63.1 & 3.3 & 50.4 & 3.0 \\
paw50 & 82.8 & 3.3 & 66.9 & 3.4 & 53.1 & 3.1 \\
paw70 & 85.5 & 4.0 & 70.8 & 3.8 & 56.5 & 3.3 \\
subc0 & 71.7 & 2.0 & 43.7 & 2.0 & 34.3 & 1.9 \\
subc30 & 81.9 & 3.1 & 63.5 & 3.2 & 50.7 & 3.0 \\
subc50 & 85.3 & 3.2 & 69.8 & 3.6 & 54.7 & 3.3 \\
subc70 & 88.0 & 4.2 & 74.5 & 4.0 & 59.8 & 3.6 \\
Abalone & 324 & 9.3 & 330 & 9.4 & 307 & 8.6 \\
breast & 45.9 & 1.7 & 25.3 & 1.4 & 21.3 & 1.4 \\
bupa & 65.1 & 2.4 & 59.1 & 2.2 & 41.5 & 2.1 \\
car-good & 26.3 & 4.0 & 27.6 & 3.8 & 26.4 & 3.9 \\
Haberman & 33.5 & 2.2 & 34.6 & 2.0 & 27 & 1.9 \\
Hepatitis & 40.8 & 1.0 & 33.1 & 0.8 & 27.2 & 1.0 \\
Pima & 176.5 & 4.9 & 167.8 & 5.1 & 115.3 & 4.7 \\
Poker & 144.6 & 6.3 & 153.2 & 6.9 & 132.7 & 6.2 \\
\hline
\end{tabular}

3) Statistical Significance: Paired t-test is one of the common ways to show that the superiority of one set of results over a second set against a set of dataset is non-random. However, in the context of classification, Demsar [35] shows the t-test suffers from a few weaknesses; the main point is related to the size of the sample, unless the two classifiers are compared on a large number of datasets or in dependant runs ( 30 datasets or more), the t-test is valid only if the differences between the two compared results are distributed normally. Therefore, assumptions of the paired t-test are not met in this study. It is important to mention here, repeating 10-cross validation 3 times on the same dataset does not satisfy the t-test condition as the runs should be independent.

Demsar [35] suggests the Wilcoxon signed-rank test as a suitable alternative to the paired t-test if the t-test conditions are not satisfied. Wilcoxon signed-rank test is a non-parametric test, hence does not require the difference in the results to be normally distributed. The test is based on the rank of the difference in the performance between the two classifiers on each dataset. Table VI shows the results of the Wilcoxon signed-rank test against the RF the base classifier. The results show that BRFA with $k=1,10$ and 20 F-Measure performance (reported in table II) is significantly better than the RF with $p$-value much smaller than 0.05 . Regarding the GM measure, the Wilcoxon test shows that only the BRAF with $k=10$ is significantly better than the RF.

TABLE VI

SHOWS THE RESULTS OF THE WILCOXON SIGNED-RANK TEST FOR BRAF WITH $k=1,10$ and 20 AGAINST THE RF THE BASE CLASSIFIER FOR EACH measure (F-Measure and Geometric Mean). P-Vale; 0.05 means THAT THE BRAF RESULTS (SHOWN IN TABLE II ) ARE SIGNIFICANTLY BETTER THAN RF.

\begin{tabular}{|c|c|c|c|c|}
\hline & & BRAF1 & BRAF10 & BRAF20 \\
\hline \multirow{5}{*}{$\sum_{i \leq}$} & W-value & 15 & 10.5 & 11.5 \\
\hline & Sum of neg. ranks & 15 & 10.5 & 11.5 \\
\hline & Sum of pos. ranks & 121 & 125.5 & 93.5 \\
\hline & Z-value & -2.7406 & -2.9733 & 2.5738 \\
\hline & p-value & 0.00614 & 0.00298 & 0.01016 \\
\hline \multirow{5}{*}{$\sum_{0}$} & W-value & 67 & 0 & 27 \\
\hline & Sum of neg. ranks & 69 & 0 & 27 \\
\hline & Sum of pos. ranks & 67 & 171 & 93 \\
\hline & Z-value & -0.0517 & -3.7236 & -1.8743 \\
\hline & p-value & 0.96012 & 0.0002 & 0.06148 \\
\hline
\end{tabular}

TABLE VII

ACCENTUATES BRAF'S IMPROVEMENT IN DIVERSITY BETWEEN TREES WHEN COMPARED AGAINST RANDOM FOREST BASE CLASSIFIER, $p=0.5$.THE BEST OVERALL RESULTS FOR EACH DATASET IS MARKED BY (*)

\begin{tabular}{lcc}
\hline & RF & BRAF \\
\hline Breast & 0.297 & $\mathbf{0 . 3 2 3}$ \\
Bupa & 0.377 & $\mathbf{0 . 4 2 0}$ \\
Car & 0.059 & $\mathbf{0 . 0 9 0}$ \\
Clover0 & 0.109 & $\mathbf{0 . 2 8 1}$ \\
Clover30 & 0.135 & $\mathbf{0 . 3 7 4}$ \\
Clover50 & 0.124 & $\mathbf{0 . 3 4 5}$ \\
clover70 & 0.144 & $\mathbf{0 . 3 6 2}$ \\
Haberman & 0.284 & $\mathbf{0 . 4 2 7}$ \\
Hepatitis & 0.188 & $\mathbf{0 . 3 7 6}$ \\
Pima & 0.308 & $\mathbf{0 . 3 8 8}$ \\
Poker & 0.027 & $\mathbf{0 . 3 4 3}$ \\
paw0 & 0.051 & $\mathbf{0 . 1 9 1}$ \\
Paw30 & 0.104 & $\mathbf{0 . 3 6 9}$ \\
paw50 & 0.096 & $\mathbf{0 . 3 5 5}$ \\
Paw70 & 0.115 & $\mathbf{0 . 4 0 6}$ \\
Subc0 & 0.046 & $\mathbf{0 . 2 2 0}$ \\
Subc30 & 0.108 & $\mathbf{0 . 3 2 8}$ \\
Subc50 & 0.113 & $\mathbf{0 . 3 1 4}$ \\
Subc70 & 0.120 & $\mathbf{0 . 3 6 8}$ \\
\hline
\end{tabular}

4) Diversity analysis: Diversity is one of the most important properties of a classifier ensemble [36]. Diversity has been used to explain the success of several ensemble methods, both in theory and in practice [28]. In addition, Wang et. al. in [37] analysed the impact of diversity on ensemble performance against the class imbalance problem.

Diversity in its basic form, is the degree to which the classifiers of each ensemble take different decisions on the same set of instances. Several methods for measuring diversity between base classifiers have been proposed [38]. One of the widely used methods is the dis-agreement measure [32] which was employed in this paper. It measures the diversity 
between individual decision trees based on the assertion that two decision trees are divergent when trained on same training set. Given two decision trees $c_{i}$ and $c_{j}$, let $n(a, b)$ be the size of the training set on which the classification decision of $c_{i}$ and $c_{j}$ is $a$ and $b$ respectively e.g. $n(1,1)$ means the number of classifiers that agreed on the same label 1 . The diversity between the two decision trees is measured by:

$$
d i s_{j, k}=\frac{n(1,-1)+n(-1,1)}{n(1,1)+n(-1,1)+n(1,-1)+n(-1,-1)}
$$

Diversity between the entire set of the decision trees is then determined by taking the average of the pairs of the decision trees.

$$
d i s=\frac{2}{L(L-1)} \sum_{j=1}^{L} \sum_{k=j+1}^{L} d i s_{j, k}
$$

Since for any two of the decision trees:

$$
n(1,1)+n(1,-1)+n(-1,1)+n(-1,-1)=N
$$

we can derive:

$$
d i s=\frac{2}{L(L-1)} \sum_{j=1}^{L} \sum_{k=j+1}^{L} n_{j, k}(-1,-1)
$$

Random forest implicitly enforces diversity by employing the random subspace algorithm to deduce splitting features from the attribute space for each root node of the individual trees [39]. BRAF boosts the already existing implicit diversity by externally generating more bootstrap samples from the difficult areas dataset, $T_{c}$. It could be logically ascertained that the bootstrap samples generated from $T_{c}$ would be different from those generated from the full dataset $T$ thereby creating diversity among the individual learning trees.

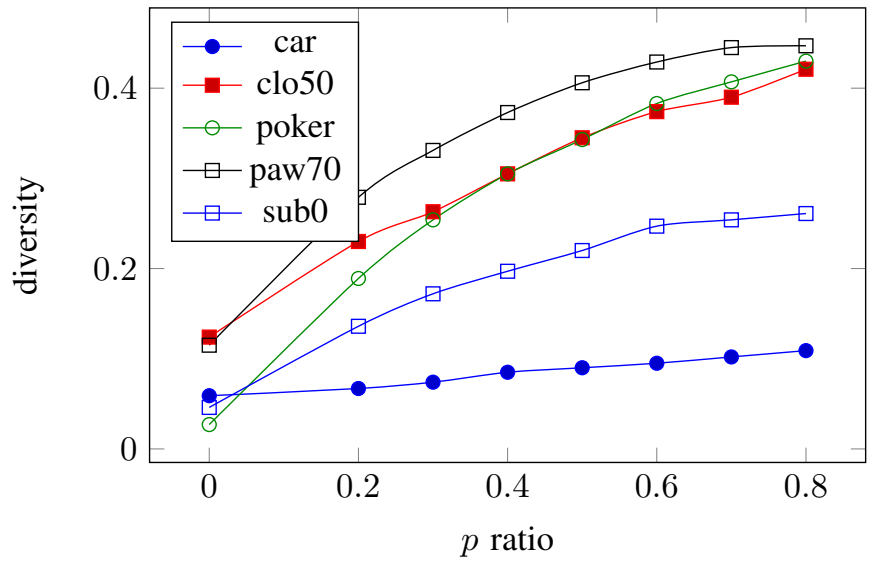

Fig. 3. shows the BRAF's diversity, the $y$ access is diversity of the assemble, the $x$ excess shows the $p$ ratio, $p=0$ is equivalent to the standard RF, while ratio $p=0.5$ means half of the trees in the assemble are generated form the difficult areas $T_{c}$

The diversity between the individual trees was measured using dis-agreement measure. Table VII shows the result of BRAF compared against the random forest base classifier.
19 datasets were used; 7 real-world application, and 12 artificial datasets. As could be deduced from Table VII, BRAF enforced higher diversity across all datasets. Also noticed, was BRAF's increase in diversity as $p$ (the parameter for defining the number of trees learning from the difficult areas, $T_{c}$ ) increases. An extension of this observation is pictorially illustrated in figure 3 shows BRAF's diversity, the $y$ access is diversity of the assemble, the $x$ excess shows the $p$ ratio, $p=0$ is equivalent to the standard $\mathrm{RF}$, while ratio $p=0.5$ means half of the trees in the ensemble are generated form the difficult areas $T_{c}$.

\section{CONCLUSION}

Class imbalance is one of the main data challenges in classification, sampling (i.e. undersampling and oversampling) is one of widely used methods which is applied on the data-level. In this paper we presented a novel, simple and effective approach for dealing with class imbalance. Rather than increasing the the minority instances in the dataset, the algorithm proposed in this paper aims to "oversample the classification ensemble" by increasing the number of classifiers that represent the minority class in the ensemble. The proposed approach could be combined with any ensemble classification method. In this study we adopted the Random Forest as it is one of the most successful and widely used classification ensemble methods. The proposed Biased Random Forest BRAF algorithm uses the the nearest neighbour algorithm, which is employed by the BRAF algorithm to identify the difficult/critical areas. Then a standard set of random forest trees is fed with more trees generated from difficult/critical areas only.

In our future work, we will investigate the relationship between the number of neighbours $k$ and the characteristics/structure of the dataset. We will look at applying BRAF concept on other ensemble classification methods. In the future we also aim to investigate to use optimisation methods to automatically discover the best performing parameters. Also, we intend to employ genetic algorithm aimed at optimizing the best $k$ value.

\section{REFERENCES}

[1] M. Galar, A. Fernandez, E. Barrenechea, H. Bustince, and F. Herrera "A review on ensembles for the class imbalance problem: bagging-, boosting-, and hybrid-based approaches," Systems, Man, and Cybernetics, Part C: Applications and Reviews, IEEE Transactions on, vol. 42, no. 4, pp. 463-484, 2012.

[2] A. Awad, M. Bader-El-Den, J. McNicholas, and J. Briggs, "Early hospital mortality prediction of intensive care unit patients using an ensemble learning approach," I. J. Medical Informatics, vol. 108, pp. 185-195, 2017. [Online]. Available: https://doi.org/10.1016/j.ijmedinf. 2017.10.002

[3] H. He, E. Garcia et al., "Learning from imbalanced data," Knowledge and Data Engineering, IEEE Transactions on, vol. 21, no. 9, pp. 12631284, 2009.

[4] V. López, A. Fernández, S. García, V. Palade, and F. Herrera, “An insight into classification with imbalanced data: Empirical results and current trends on using data intrinsic characteristics," Information Sciences, vol. 250, pp. 113-141, 2013.

[5] B. W. Yap, K. A. Rani, H. A. A. Rahman, S. Fong, Z. Khairudin, and N. N. Abdullah, "An application of oversampling, undersampling, bagging and boosting in handling imbalanced datasets," in Proceedings of the First International Conference on Advanced Data and Information Engineering (DaEng-2013). Springer, 2014, pp. 13-22. 
[6] K. Napierała, "Improving rule classifiers for imbalanced data," 2012.

[7] N. Japkowicz, C. Myers, M. Gluck et al., "A novelty detection approach to classification," in IJCAI, 1995, pp. 518-523.

[8] R. Barandela, J. S. Sánchez, V. Garcia, and E. Rangel, "Strategies for learning in class imbalance problems," Pattern Recognition, vol. 36, no. 3, pp. 849-851, 2003

[9] M. Bader-El-Den, "Self-adaptive heterogeneous random forest," in Computer Systems and Applications (AICCSA), 2014 IEEE/ACS 11th International Conference on. IEEE, 2014, pp. 640-646.

[10] T. Perry, M. Bader-El-Den, and S. Cooper, "Imbalanced classification using genetically optimized cost sensitive classifiers," in IEEE Congress on Evolutionary Computation (CEC). IEEE, 2015, pp. 680-687.

[11] N. V. Chawla, K. W. Bowyer, L. O. Hall, and W. P. Kegelmeyer, "Smote: synthetic minority over-sampling technique," Journal of artificial intelligence research, pp. 321-357, 2002.

[12] H. Han, W.-Y. Wang, and B.-H. Mao, "Borderline-smote: a new oversampling method in imbalanced data sets learning," in Advances in intelligent computing. Springer, 2005, pp. 878-887.

[13] T. Maciejewski and J. Stefanowski, "Local neighbourhood extension of smote for mining imbalanced data," in IEEE Symposium on Computational Intelligence and Data Mining (CIDM). IEEE, 2011, pp. 104-111.

[14] C. Bunkhumpornpat, K. Sinapiromsaran, and C. Lursinsap, "Safelevel-smote: Safe-level-synthetic minority over-sampling technique for handling the class imbalanced problem," in Advances in Knowledge Discovery and Data Mining. Springer, 2009, pp. 475-482.

[15] H. He, Y. Bai, E. A. Garcia, and S. Li, "Adasyn: Adaptive synthetic sampling approach for imbalanced learning," in Neural Networks, 2008. IJCNN 2008.(IEEE World Congress on Computational Intelligence). IEEE International Joint Conference on. IEEE, 2008, pp. 1322-1328.

[16] D. L. Wilson, "Asymptotic properties of nearest neighbor rules using edited data," Systems, Man and Cybernetics, IEEE Transactions on, no. 3 , pp. $408-421,1972$.

[17] J. Laurikkala, Improving identification of difficult small classes by balancing class distribution. Springer, 2001.

[18] J. R. Quinlan, "Bagging, boosting, and c4. 5," in AAAI/IAAI, Vol. 1, 1996, pp. 725-730.

[19] R. E. Schapire and Y. Freund, Boosting: Foundations and algorithms. MIT press, 2012

[20] J. Zhu, H. Zou, S. Rosset, and T. Hastie, "Multi-class adaboost," Statistics and its Interface, vol. 2, no. 3, pp. 349-360, 2009.

[21] L. Breiman, "Random forests," Machine learning, vol. 45, no. 1, pp. 5-32, 2001.

[22] A. Liaw and M. Wiener, "Classification and regression by randomforest," $R$ news, vol. 2, no. 3, pp. 18-22, 2002.

[23] C. Chen, A. Liaw, and L. Breiman, "Using random forest to learn imbalanced data," University of California, Berkeley, pp. 1-12, 2004.

[24] S. Wang and X. Yao, "Diversity analysis on imbalanced data sets by using ensemble models," in IEEE Symposium on Computational Intelligence and Data Mining (CIDM). IEEE, 2009, pp. 324-331.

[25] E. Y. Chang, B. Li, G. Wu, and K. Goh, "Statistical learning for effective visual information retrieval." in ICIP (3). Citeseer, 2003, pp. 609-612.

[26] D. Tao, X. Tang, X. Li, and X. Wu, "Asymmetric bagging and random subspace for support vector machines-based relevance feedback in image retrieval," IEEE transactions on pattern analysis and machine intelligence, vol. 28, no. 7, pp. 1088-1099, 2006.

[27] S. Hido, H. Kashima, and Y. Takahashi, "Roughly balanced bagging for imbalanced data," Statistical Analysis and Data Mining, vol. 2, no. 5-6, pp. 412-426, 2009.

[28] R. Polikar, "Ensemble based systems in decision making," IEEE Circuits and systems magazine, vol. 6, no. 3, pp. 21-45, 2006.

[29] J. Alcalá, A. Fernández, J. Luengo, J. Derrac, S. García, L. Sánchez, and F. Herrera, "Keel data-mining software tool: Data set repository, integration of algorithms and experimental analysis framework," Journal of Multiple-Valued Logic and Soft Computing, vol. 17, no. 2-3, pp. 255287, 2010

[30] C. Blake and C. J. Merz, " $\{\mathrm{UCI}\}$ repository of machine learning databases," 1998.

[31] J. Stefanowski, "Overlapping, rare examples and class decomposition in learning classifiers from imbalanced data," in Emerging Paradigms in Machine Learning. Springer, 2013, pp. 277-306.

[32] D. B. Skalak, "The sources of increased accuracy for two proposed boosting algorithms," in Proc. American Association for Artificial Intelligence, AAAI-96, Integrating Multiple Learned Models Workshop, vol. 1129. Citeseer, 1996, p. 1133

[33] M. Bader-El-Den, E. Teitei, and M. Adda, "Hierarchical classification for dealing with the class imbalance problem," 2016.
[34] H. Hoos and K. Leyton-Brown, "An efficient approach for assessing hyperparameter importance," in International Conference on Machine Learning, 2014, pp. 754-762

[35] J. Demšar, "Statistical comparisons of classifiers over multiple data sets," Journal of Machine learning research, vol. 7, no. Jan, pp. 1-30, 2006.

[36] L. Kuncheva, M. Skurichina, and R. P. Duin, "An experimental study on diversity for bagging and boosting with linear classifiers," Information fusion, vol. 3, no. 4, pp. 245-258, 2002.

[37] S. Wang and X. Yao, "Relationships between diversity of classification ensembles and single-class performance measures," IEEE Transactions on Knowledge and Data Engineering, vol. 25, no. 1, pp. 206-219, 2013.

[38] E. K. Tang, P. N. Suganthan, and X. Yao, "An analysis of diversity measures," Machine Learning, vol. 65, no. 1, pp. 247-271, 2006.

[39] K. Fawagreh, M. M. Gaber, and E. Elyan, "Diversified random forests using random subspaces," in International Conference on Intelligent Data Engineering and Automated Learning. Springer, 2014, pp. 85-92.

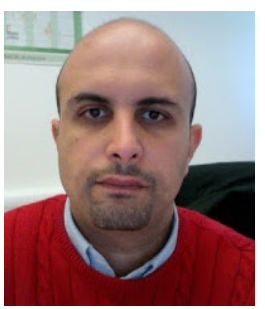

Mohamed Bader-El-Den Received the BS and MSc degree in computer engineering from the Arab Academy for Science and Technology, Alexandria, Egypt in 2000 and 2003 respectively, and $\mathrm{PhD}$ degree in computer science from the University of Essex, UK in 2009, during his $\mathrm{PhD}$ he did an internship at Microsoft Research Cambridge. He is currently a senior lecturer at the University of Portsmouth, UK, where he is a member of the computational intelligence group. Previously, he was a research associate at the Loughborough University. His current research interests include data mining, classification, class imbalance, evolutionary computation, genetic programming, Combinatorial optimisation and big data. On the professional level, he worked as Visual C++ developer for three years at Harf for information technology, Egypt. Also, Also, he was Sun Certified Programmer and Oracle Database Administration Certified (OCP-DBA).

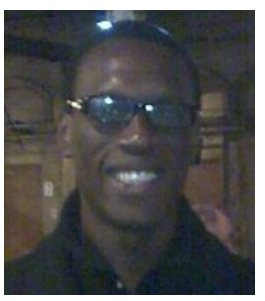

Eleman Teitei received his BSc in Computer Science and MSc in Database Systems from the Niger Delta University, Nigeria and University of Central Lancashire, UK in 2007 and 2012 respectively. He is currently a $\mathrm{PhD}$ in Computing student at the University of Portsmouth, U.K. He was previously a lecturer at the Bayelsa State College of Arts and Science, Nigeria. His research area is in data mining and machine learning with focus on the tackling the class imbalance problem in supervised learning. $\mathrm{He}$ is also a Sun Certified Java Programmer, a Sun Certified Web Component Developer, a Certified Associate in Project Management, an Oracle Certified Administrator and also a member of the Project Management Institute, U.S.A.

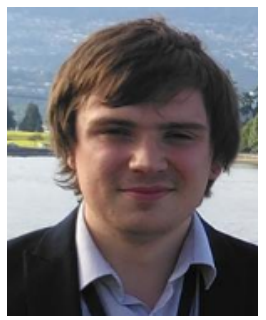

Todd Perry received his BSc in Computer Science from the school of computing, University of Portsmouth, UK. Currently he works as a Software Engineer at Huq Industries, a market intelligence company based in London, UK. Prior to that he was researcher at the research and development department at Lockheed Martin, UK where he was involved involved in several data mining projects. His current research interests include machine learning, big data classification, class imbalance, evolutionary computation, Combinatorial optimisation. 